\title{
Estudo das variáveis que contribuem para o nível de empatia nos acadêmicos de medicina
}

\author{
Natália da S. Fontana, ${ }^{1}$ Ana Amélia F. Vilela, ${ }^{2}$ Aridiane A. Ribeiro, ${ }^{2}$ Verônica C. Ferreira, ${ }^{2}$ Geovana M. \\ Peres, ${ }^{1}$ Mathias R. Macedo, ${ }^{1}$ Nataly C. S. e Souza, ${ }^{1}$ Niulane R. Carrijo, ${ }^{1}$ Sanmer J. S. Ferreira, ${ }^{1}$ \\ Adriana A. Carvalho ${ }^{2 *}$
}

\begin{abstract}
Resumo
Introdução: Empatia é definida como processo psicológico orientado por mecanismos que englobam as esferas afetiva, cognitiva e comportamental quanto à observação da experiência do outro, essencial à área médica no estabelecimento de confiança entre médico e paciente. Objetivos: Identificar as variáveis que podem influenciar nos níveis de empatia no decorrer da graduação médica, tais como gênero, ano de graduação e escolha da especialidade médica. Materiais e Métodos: Realizou-se consulta nos bancos de dados: BIREME, PubMed e Google Scholar. Os critérios de inclusão na seleção foram os idiomas, período de publicação (2007-2018), e que correspondessem à temática, e exclusão de artigos que avaliavam os índices de empatia com profissionais e alunos de residência médica. Resultados: Os resultados foram diversificados, desde a afirmação de que o grau de empatia não alterava com o passar dos anos na graduação de medicina até que esse nível se modificava entre o primeiro e sexto ano entre os estudantes, em que prevalecera a notável queda dos níveis de empatia após o terceiro ano da graduação, significativamente no sexo masculino. Conclusões: Observa-se que durante a graduação em cursos de medicina há uma redução dos níveis de empatia, durante o ciclo clínico, algo que afeta negativamente a relação médico-paciente, promovendo dificuldades na comunicação e na adesão ao tratamento.
\end{abstract}

Descritores: Medicina; Centros Médicos Acadêmicos; Empatia.

\section{Abstract \\ Study of the variables that contribute to the level of empathy in medical students}

Introduction: Empathy is defined as a psychological process guided by mechanisms that encompass the affective, cognitive and behavioral spheres regarding the observation of the other's experience, essential to the medical field in establishing trust between doctor and patient. Objectives: To identify the variables that can influence the levels of empathy during medical graduation, such as gender, year of graduation and choice of medical specialty. Materials and Methods: The databases: BIREME, PubMed and Google Scholar were consulted for the review. The criteria for inclusion were the languages, period of publication (2007-2018), and thematic match, and exclusion of articles that evaluated the rates of empathy with professionals and students of medical residency. Results: Results were diversified, from the claim that the degree of empathy did
1. Graduação em medicina, Universidade Federal de Jataí, Jataí, Goiás, Brasil.

2. Unidade Acadêmica Especial Ciências da Saúde, Universidade Federal de Jataí, Jataí, Goiás, Brasil.

*Endereço para correspondência:

BR 364, Km 195, no 3800

Campus Jatobá, Cidade Universitária.

Jataí, GO, CEP: 75801-615

E-mail: adriana.assis@ufg.br

BJHBS, Rio de Janeiro, 2020;19(1):57-62

Received on 01/13/2020. Approved on 04/17/2020.

not change over the years in medical school until that level changed between the first and sixth year among students, in which the notable fall in empathy levels prevailed after the third year of graduation, significantly in males. Conclusions: It was observed that during graduation in medical courses there is a reduction in empathy levels, during the clinical cycle, something that negatively affects the doctor-patient relationship, promoting difficulties in communication and patient adherence to treatment.

Keywords: Medicine; Academic Medical Center; Empathy.

\section{Resumen}

\section{Estudio de los variables que contribuyen al nivel} de empathy en estudiantes medicales

Introdución: La empatía se define como un proceso psicológico guiado por mecanismos que abarcan las esferas afectiva, cognitiva y conductual con respecto a la observación de la experiencia del otro, esencial para el campo médico en el establecimiento de la confianza entre el médico y el paciente. Objetivo: Identificar las variables que pueden influir en los niveles de empatía durante la graduación médica, como el género, el año de graduación y la elección de la especialidad médica. Materiales y Métodos: La consulta se realizó en las bases de datos: BIREME, PubMed y Google Scholar. Los criterios para la inclusión en la selección fueron los idiomas, el período de publicación (2007-2018), que correspondía al tema, y la exclusión de los artículos que evaluaban las tasas de empatía con los profesionales y estudiantes de residencia médica. Resultados: Los resultados fueron diversos. Desde la 


\section{Artigo de revisão}

afirmación de que el grado de empatía no cambió con los años en la escuela de medicina hasta que ese nivel cambió entre el primer y sexto año entre los estudiantes, en el que prevaleció la notable disminución en los niveles de empatía después del tercero. año de graduación, significativamente en varones. Conclusiones: Se observa que durante los

\section{Introdução}

A empatia é um dos símbolos das profissões da área da saúde, ${ }^{1}$ sendo assunto recorrente em diversos estudos e pesquisas, que avaliam sua importância durante a formação médica e na construção da relação médico-paciente. A empatia é definida por Davis, ${ }^{2}$ como um processo psicológico orientado por mecanismos que englobam as esferas afetiva, cognitiva e comportamental quanto à observação da experiência do outro. Esse conceito é trabalhado por Rogers, ${ }^{3}$ que o caracteriza como um processo de sensibilização pelas mudanças sentidas e demonstradas pelo indivíduo.

Juugärvi e colaboradores ${ }^{4}$ e Lampert e colaboradores ${ }^{5}$ reforçam que a empatia está estritamente ligada à responsabilidade das pessoas diante de seus relacionamentos. Ela pode ser descrita pelo ato da compreensão e abstração do sentir e o viver do paciente, e, por isso, torna-se instrumento primordial na área médica para o estabelecimento de confiança mútua entre o médico e seu paciente, permitindo assim um espaço seguro e confortável para a comunicação entre ambas às partes e, consequentemente, permite o exercício de uma medicina de excelência. Porto ${ }^{6}$ considera uma medicina de excelência aquela que adiciona ao exame clínico as qualidades humanas, princípios éticos e a relação médico-paciente.

Tem-se observado que durante a graduação em cursos de medicina há uma redução dos níveis de empatia, o que afeta negativamente a relação médicopaciente e tem como consequências dificuldades na comunicação e prejuízos na adesão ao tratamento. $\mathrm{O}$ estudo realizado até o momento demonstra que os índices relacionados à empatia tendem a diminuir ao longo do terceiro ano de medicina, período esse do curso em que os acadêmicos começam a ter contato com os primeiros pacientes na clínica médica especialidades nas faculdades e universidades do Brasil e do mundo. ${ }^{?}$

\section{Objetivo}

Identificar as variáveis que podem influenciar nos níveis de empatia no decorrer da graduação médica, tais como gênero, ano de graduação e escolha da especialidade médica. cursos de pregrado de medicina hay una reducción en los niveles de empatía durante el ciclo clínico, algo que afecta negativamente la relación médico-paciente, promoviendo dificultades de comunicación y adherencia al tratamiento.

Palabras clave: Medicina; Centros Médicos Académicos; Empatía.

\section{Metodologia}

Este estudo constitui-se de uma revisão integrativa da literatura. A busca nos bancos de dados foi realizada na BIREME, PubMed e Google Scholar, durante o período de outubro de 2016 a abril de 2018, utilizando os descritores cadastrados nos Descritores em Ciências da Saúde criados pela Biblioteca Virtual em Saúde (BVS), as quais foram: medicina, estudantes, acadêmicos, empatia e suas respectivas traduções para a língua inglesa (medicine, students, academic, empathy).

Os critérios de inclusão para a seleção dos artigos foram os idiomas, período de publicação, trabalhos originais e que apresentassem adequação à temática. Foram selecionados artigos em português, inglês e espanhol; foi feito um corte cronológico de estudos publicados entre 2007 a 2018; publicações que versavam sobre os estudantes de medicina (matriculados em instituições públicas e privadas ao longo do curso de graduação), e publicações que avaliassem a influência do gênero, ano de graduação e escolha da especialidade médica no nível de empatia. Foram excluídos artigos que avaliavam os índices de empatia em profissionais e alunos de residência médica (Figura 1).

\section{Resultados e discussão}

Foram encontrados, no total, 262 trabalhos. Os critérios de seleção foram feitos a partir da leitura do título, leitura do resumo e, por fim, a leitura do artigo. Dessa forma, foram selecionados 74 artigos originais que estavam distribuídos nos seguintes bancos de dados: BIREME (37), PubMed (25) e Google Scholar (12).

$\mathrm{O}$ ano de maior publicação de trabalhos sobre empatia na relação médico-paciente foi em 2017 com 18 artigos, seguido de 2014 (14), 2016 (13), 2013 (12), 2015 (7), 2012 (7), 2018 (3). Com relação à característica metodológica foram encontrados 58 estudos transversais e 18 longitudinais.

Embora o maior número de publicações encontradas tenha sido de trabalhos que utilizaram estudo transversal, Magalhães e colaboradores, ${ }^{8}$ Youssef e colaboradores, ${ }^{9}$ Tariq $^{10}$ e Yang Song ${ }^{11}$ apontaram que este tipo de pesquisa não permite fazer relações causais entre o nível de empatia e as variáveis (gênero, ano 


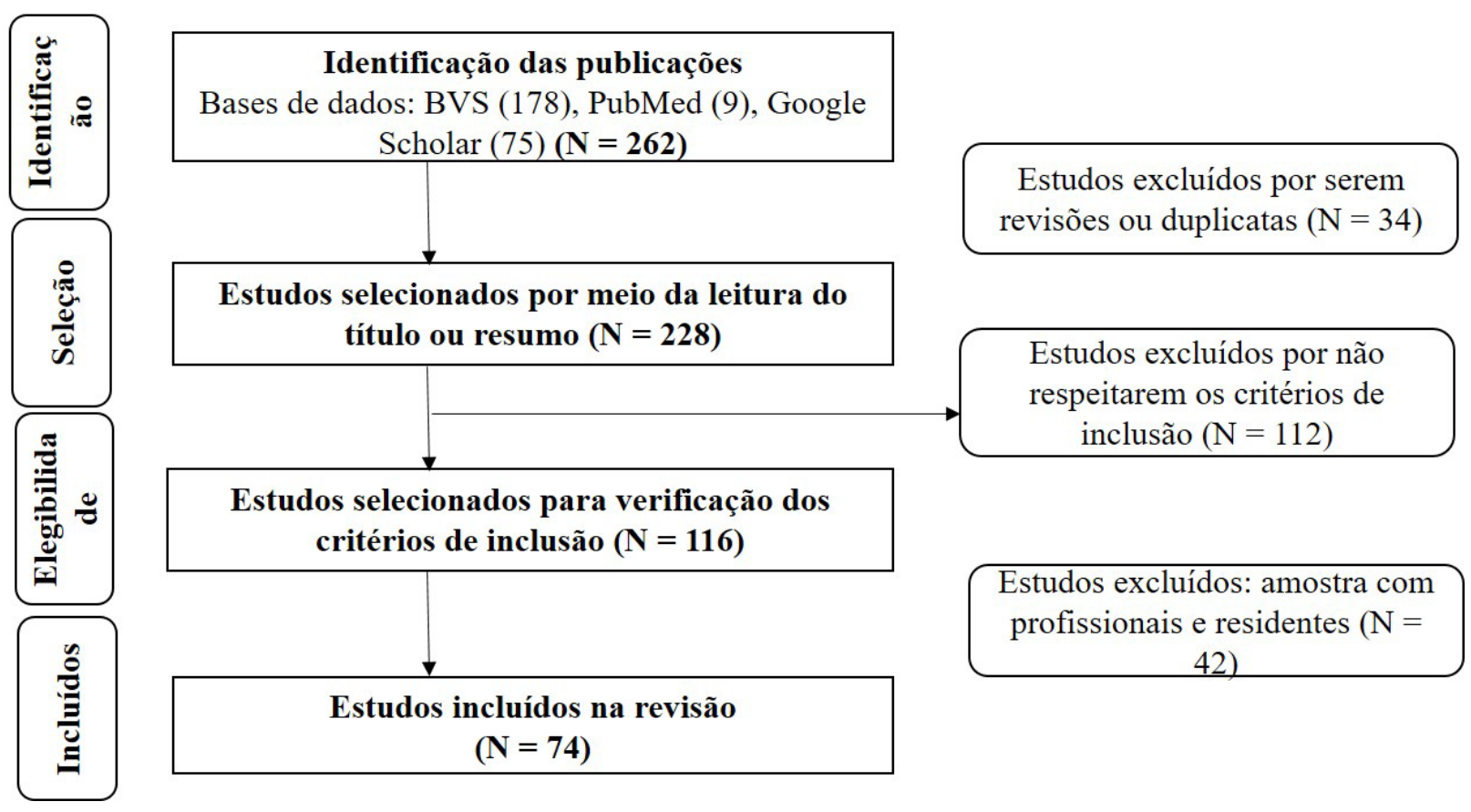

Figura 1. Fluxograma da seleção dos estudos

de graduação, escolha da especialidade). Estes autores recomendam a realização de estudos longitudinais para estabelecer a causalidade.

Dos 74 artigos analisados, a Escala Jefferson de Empatia Médica foi o instrumento mais prevalente para avaliar a empatia entre estudantes de medicina, correspondendo à 35\% (26) do total, o que corrobora a validade do instrumento em nível mundial, como também sua utilização e tradução para variados idiomas. A versão adaptada para estudantes (JSPE-S) foi encontrada em 25 trabalhos. A Escala Jefferson de Empatia Médica, contem 20 questões e foi criada com a necessidade de estudiosos da Jefferson Medical College em construir um instrumento para avaliar a empatia no contexto do ensino médico. ${ }^{12}$ Paro e colaboradores ${ }^{13}$ validou esta escala no Brasil.

Outros instrumentos também foram utilizados entre eles o Balanced Emotional Empathy Scale (BEES), Pencil and Paper Empathy Ratin, Social Empathy Index, Big Five Inventory (BFI), além de trabalhos realizados associando mais de um instrumento. Além destes, o Interpersonal Reactivity Index (IRI) e a Escala Multidimensional de Reatividade Interpessoal de Davis (EMRI). Koller e colaboradores ${ }^{14}$ adaptaram e validaram o IRI para o contexto brasileiro, ficando conhecida como Escala Multidimensional de Reatividade Interpessoal (EMRI). ${ }^{15}$
Os estudos selecionados foram realizados, em sua maioria, no Brasil (13), seguido dos Estados Unidos da América (10) e Portugal (7). Foram encontrados também trabalhos desenvolvidos na Colômbia (4), Coreia doSul (4), China (2), Alemanha (2), Irã (2), Iraque (1), Inglaterra (1), Irlanda (1), Etiópia (1), Austrália (1), entre outros. O interesse pelo estudo da empatia médica tem crescido devido a sua influência nos desfechos clínicos (maior precisão no diagnóstico e prognóstico). Quando a empatia está presente na relação médico-paciente este último tende a seguir as orientações terapêuticas resultando no controle da doença já instalada. ${ }^{12,16-19}$

A queda progressiva do nível de empatia ao longo dos anos na formação médica foi descrita por Magalhães e colaboradores, ${ }^{8}$ Stratton, Saunders e Elam ${ }^{20}$ e Hojat e colaboradores. ${ }^{12}$ Nossos achados confirmam essa afirmativa visto que dos 43 trabalhos que estudaram a relação entre o nível de empatia e o ano de graduação, 27 estudos (63\%) demonstraram declínio da empatia ao longo dos anos enquanto 16 estudos (37\%) não houve declínio.

Os estudos de Silva ${ }^{21}$ apresentaram um declínio gradual da empatia, com picos maiores no terceiro e principalmente no sexto ano, que segundo ele se deve ao início do ciclo clínico e o estresse do mercado de trabalho e provas de residência, respectivamente. Por outro lado, Magalhães e colaboradores ${ }^{8}$ e Mostafa e 
colaboradores ${ }^{22}$ avaliaram alunos do primeiro e sexto períodos, e encontraram que os alunos que estavam no início do curso tiveram menores índices de empatia do que os que estavam no sexto período. Os resultados divergentes entre a associação do nível de empatia e o ano de graduação demonstra a necessidade de realizar um estudo longitudinal para averiguar a relação causal entre as variáveis..$^{8-11,23}$

Em relação a associação do nível de empatia com o gênero, encontramos 42 trabalhos que fizeram tal associação, nos quais 26 demonstraram associação positiva entre o nível de empatia e o gênero. Os estudos apontam que as mulheres possuem maior empatia quando comparadas aos homens. ${ }^{8,24,25}$ Dentre os motivos mencionados como justificativas para os dados encontrados, Rahimi-Madiseh e colaboradores ${ }^{24}$ evidenciaram a existência de uma forte concepção cultural no Irã, na qual há papéis socialmente estritos e demarcados para cada gênero. As mulheres seriam responsáveis por cuidar do ambiente doméstico e de seus familiares, e, portanto, um papel mais empático em relação ao seu próximo, enquanto que o homem, por exercer papéis primordiais como o de provedor financeiro e chefe da família, teria uma característica pouco empática. Magalhães e colaboradores ${ }^{8}$ relataram que as mulheres possuem maior capacidade de desenvolverem um sentimento mais forte ao cuidar da prole do que os homens, e, por isso, são mais hábeis na compreensão e comunicação com seus descendentes, além de serem mais propensas a desenvolver relações interpessoais, oferecer apoio emocional, sensibilidade e cuidado do que os homens.

Dentre os estudos que não registraram uma alteração significativa nos níveis de empatia entre o gênero, Paro e colaboradores ${ }^{13}$ mencionam que uma explicação plausível para esse registro seria a amostragem utilizada no estudo, uma vez que significativa parte dos acadêmicos participantes não relataram seu sexo.

Quando o nível de empatia foi associado a escolha da especialidade médica, dos 47 trabalhos selecionados, 9 estudaram essa relação. Os trabalhos apontaram maior nível de empatia nos estudantes que tinham a pretensão de se especializar em medicina da família ou psiquiatria, e o menor nível de empatia estava relacionado com a escolha de cirurgia, radiologia e patologia. Santos e colaboradores ${ }^{26}$ afirmaram que os estudantes com preferência em uma especialidade orientada para as pessoas possuem maior nível de empatia quando comparada aos estudantes que preferem alguma especialidade voltada para a tecnologia.

Diante do exposto, evidenciam-se a necessidade de implementação de métodos e de estratégias que visem o desenvolvimento e/ou o fortalecimento da empatia durante a graduação de medicina, tais como ensino voltado para uma formação mais humanista, independentemente da especialidade, e que tal abordagem se intensifique nos últimos períodos do curso, nos quais há maior interação com a prática clínica e o exercício da relação médico-paciente.8,28

Entre os artigos selecionados, dois estudos mencionaram e descreveram em seus resultados, estratégias e meios adotados para a melhora da empatia nos acadêmicos durante o período de graduação médica. ${ }^{27,28}$

Os acadêmicos participantes do terceiro ano de seu estudo, e que foram submetidos a sessões interativas intituladas "Humanismo e Profissionalismo" durante a passagem pelas especialidades clínicas, preservaram o nível da empatia e demonstraram mais capazes de reconhecer modelos positivos e negativos de cuidado ao paciente, apresentando maior entusiasmo, altruísmo e preocupação para com seus pacientes. ${ }^{28}$

Ainda foram utilizados blogs anônimos e artigos para iniciar a discussão sobre o medo do fracasso e da culpa, e sobre a responsabilidade e apreço pelas informações confidencializadas ao profissional médico pelo paciente. Muitos alunos reconheceram insegurança em seus próprios conhecimentos e habilidades, e alívio pela oportunidade de discutir sentimentos como esses, e de admitirem seus próprios erros, como também de observarem os erros de seus colegas em situações semelhantes. ${ }^{28}$

Os autores concluíram que é de grande importância a existência no currículo do terceiro ano de graduação de medicina de espaços e horários reservados para o compartilhamento de sentimentos, e para o desenvolvimento e/ou validação de um comportamento mais humanista entre os acadêmicos, com o intuito de manter, ou até mesmo aumentar, seus níveis de empatia ao longo do curso. ${ }^{28}$

Já Magalhães e colaboradores ${ }^{8}$ relataram que o aumento registrado na empatia entre os acadêmicos, embora possam ser efeitos da coorte, refletem também a influência da ênfase curricular nos princípios de humanismo e centralização do paciente na assistência médica, que já se inicia nas quatro primeiras semanas da graduação, além de apresentar um programa de humanidades que se mantém do primeiro ao sexto ano do curso, o qual permite a manutenção e o treinamento 
de habilidades empáticas e de comunicação nos futuros profissionais médicos.

\section{Conclusão}

Esta revisão bibliográfica buscou identificar os fatores que influenciam no grau de empatia dos acadêmicos de medicina ao longo do curso (tais como: período do curso e sexo/gênero), uma vez que a redução no grau de empatia nos acadêmicos de medicina interfere negativamente no estabelecimento, fortalecimento e qualidade da relação médico-paciente, e, consequentemente, no exercício da medicina de qualidade.

Em relação à diferença no nível de empatia entre os gêneros dos estudantes, sendo essa superior nas mulheres, a justificativa apresentada por parte dos estudos de Rahimi-Madiseh e colaboradores ${ }^{24}$ e de Magalhães e colaboradores ${ }^{8}$ reafirmam o consagrado contexto cultural e social dos papéis desempenhados pelas mulheres e pelos homens na sociedade, evidenciando o papel natural de cuidado da prole e de seus familiares pelas mulheres, o que explicaria a maior capacidade e habilidade empática dessas com seu próximo, e, portanto, com seu paciente.

Embora a maioria dos artigos analisados apresentarem redução no nível de empatia entre os acadêmicos de medicina, durante o ciclo clínico, alguns trazem melhora significativa da empatia devido a inserção em seu currículo de programas que visem o treinamento das habilidades humanísticas, como sessões interativas intituladas "Humanismo e Profissionalismo", proporcionando, assim, aperfeiçoamento na relação médico-paciente e maior cuidado com o paciente.

\section{Referências}

1. Larson BE, Yao X. Clinical empathy as emotional labor in the patient-physician relationship: empathy, emotional labor and acting. JAMA. 2005 Mar 2; 293(9):1100-6.

2. Davis MH. Empathy: a social psychological approach. Boulder: Westview Press; 1996. 260 p.

3. Rogers CR. The necessary and sufficient conditions for therapeutic personality change. J Consult Clin Psychol. 1957;21(2):95-103.

4. Juujärvi S, Myyry L, Pesso K. Empathy and values as predictors of care development. Scand J Psychol. 2012 Aug 07;53(5):413-20.

5. Lampert CDT, Scortegagna SA. Empatia em cuidadores de idosos por meio do Teste Pfister. Arq Bras Psicol. 2018 Oct 25;70(1):193-205.

6. Porto CC. Cartas aos estudantes de medicina. Rio de Janeiro: Guanabara Koogan, 2014. 320 p.

7. Chen D, Lew R, Hershman W, et al. A cross-sectional meas- urement of medical student empathy. J Gen Intern Med. 2007 Oct;22(10):1434-8.

8. Magalhães E, Salgueira AP, Costa P, Costa M. J. Empathy in senior year and first year medical students: a cross-sectional study. BMC Med Educ. 2011;11(52).

9. Youssef FF, Nunes P, Sa B, et al. An exploration of changes in cognitive and emotional empathy among medical students in the Caribbean. Int J Med Educ. 2014;5:185-92.

10. Tariq N, Rasheed T, Tavakol M. A quantitative study of empathy in pakistani medical students: a multicentered approach. J Prim Care Community Health. 2017 Oct;8(4):294-9.

11. Song Y, Shi M. Associations between empathy and big five personality traits among Chinese undergraduate medical students. PLoS One. 2017 Feb;12(2):e0171665.

12. Hojat M, Mangione S, Nasca TJ, et al. An empirical study of decline in empathy in medical school. Med Educ. 2004 Sep;38(9):934-41.

13. Paro HBMS, Daud-Gallotti RM, Tibério IC, et al. Brazilian version of the Jefferson Scale of Empathy: psychometric properties and factor analysis. BMC Med Educ. 2012;12(73).

14. Koller SH, Camino C, Ribeiro J. Adaptação e validação de duas escalas de empatia para uso no Brasil. Estudos de Psicologia. 2001 Dec;18(3):43-53.

15. Sampaio LR, Guimarães PRB, Camino CPS, et al. Estudos sobre a dimensionalidade da empatia: tradução e adaptação do Interpersonal Reactivity Index (IRI). PSICO PUCRS. 2011;42(1):67-76.

16. Provenzano BC, Machado APG, Rangel M, et al. A empatia médica e a graduação em medicina. Rev HUPE. 2014 OctDec;13(4):19-25.

17. Hojat M. Empathy in patient care: antecedents, development, measurement, and outcomes. New York: Springer; 2007. 296 p.

18. Barski AJ. Hidden reasons some patients visit doctors. Ann Int Med. 1981 Apr;94(4 pt 1):492-8.

19. Dubnicki C. Relationships among therapist empathy and authoritarianism and a therapist's prognosis. J Consult Clin Psychol. 1977 Oct;45(5):958-9.

20. Stratton TD, Saunders JA, Elam CL. Changes in medical students' emotional intelligence: an exploratory study. Teach Learn Med. 2008 Jul;20(3):279-84.

21. Silva AL. No coração da escolar médica: um estudo preliminar da empatia na formação médica [dissertação]. [Porto Alegre]: Pontifícia Universidade Católica do Rio Grande do Sul; 2015. $66 \mathrm{p}$.

22. Mostafa A, Hoque R, Mostafa MD, et al. Empathy in undergraduate medical students of Bangladesh: psychometric analysis and differences by gender, academic year, and specialty preferences. ISRN Psychiatry. 2014 Feb;375439.

23. Chatterjee A, Ravikumar R, Singh S, et al. Clinical empathy in medical students in India measured using the Jefferson Scale of Empathy-Student Version. J Educ Eval Health Prof. 2017 Dec 27;14:33.

24. Rahimi-Madiseh, M, Tavakol M, Dennick R, et al. Empathy in Iranian medical students: a preliminary psychometric analysis and differences by gender and year of medical school. Med Teacher. 2010;32(11):e471-8.

25. Shariat SV, Habibi M. Empathy in Iranian medical students: measurement model of the Jefferson Scale of Empathy. Med Teacher. 2013;35(1):e913-8.

26. Santos MA, Grosseman S, Morelli TC, et al. Empathy diferences by gender and speciality preference in medical students: a study in Brazil. Int J Med Educ. 2016 May 21; 7:149-53. 


\section{Artigo de revisão}

27. LoSasso AA, Lamberton C, Sammon MB, et al. Enhancing student empathetic engagement, history-taking, and communication skills during electronic medical record use in patient care. Acad Med. 2017 Jul;92(7):1022-7.

28. Rosenthal S, Howard B, Schlussel YR, et al. Humanism at heart: preserving empathy in third-year medical students. Acad Med. 2011 Mar;86(3):350-8.

40. Stefanaki C, Peppa M, Boschiero D, et al. Healthy overweight/ obese: early osteosarcopenic obesity features. Eur J Clin Invest. 2016;46(9):767-778. 